\title{
Inter-Carrier Interference Analysis of OFDM Systems Communicating Over Rapidly-Fading Nakagami- $m$ Channels
}

\author{
Jianmin Zhang, Lie-Liang Yang, Xiang Liu and Lajos Hanzo \\ School of ECS, University of Southampton, SO17 1BJ, UK.
}

\begin{abstract}
In this contribution, the Bit Error Rate (BER) performance of OFDM systems using coherent demodulation over time-variant flat Nakagami- $m$ fading channels is analyzed. In a rapidly fading environment the orthogonality of the OFDM subcarrier signals may be destroyed and this phenomenon potentially results in InterCarrier Interference (ICI). The novel contribution of the paper is that the desired signal is shown to be accurately modelled as a function of the sum of two correlated Nakagami$m$ variables, provided that the Doppler shift is moderate. It is also shown that the ICI can be modelled as a function of the difference of two correlated Nakagami- $m$ variables. Finally, it is demonstrated that the assumption of having a near-Gaussian ICI distribution is inaccurate, hence leading to an inaccurate BER estimate, which is associated with a pessimistically high BER-floor.
\end{abstract}

\section{INTRODUCTION}

In recent years Orthogonal Frequency Division Multiplexing (OFDM) has emerged as promising candidates for next generation communications systems [1]. The Bit Error Rate (BER) performance of OFDM systems communicating over Rayleigh fading channels has been extensively studied. These contributions usually assumed that the fading channel's envelope is constant within an OFDM symbol. However, in case of a high carrier frequency, high mobile speed and long OFDM symbols, the channel envelope fluctuation across an OFDM symbol cannot be neglected. This results in InterCarrier Interference (ICI) owing to the loss of orthogonality across the OFDM subcarriers and hence degrades the achievable BER performance. To elaborate a little further, the BER attained in non-stationary Rayleigh fading channels has been investigated in [1]-[6]. The BER performance of a plethora of sophisticated schemes has been studied using simulations in [1], [2]. By contrast, references [3]-[4] provided analytic BER performance expressions based on the assumption that the ICI may be approximated by an additive Gaussian distribution. However, as we will demonstrate in this contribution, this assumption is inaccurate and results in a pessimistic error floor. Furthermore, Bradaric and Petropulu [5] studied the achievable BER performance using the joint distribution of the time-invariant channel and the time-variant component of the channel. Wang, Proakis and Zeidler [6] presented a BER formula derived by exploiting the analytical Probability

The financial support of the Royal Society Sino-British Fellowship Trust Award is gratefully acknowledged.
Density Function (PDF) of the ICI, invoking an approximation technique referred to as the Gram-Charlier series.

Although historically Rayleigh fading channels have been used in the analysis of wireless communications systems, recently the Nakagami- $m$ fading channel model has drawn considerable attention [7] since it not only entails both the Rayleigh and Ricean distribution in a convenient joint framework but also matches a range of experimental propogation data. Against this background, the novel contribution of this paper is that the BER performance of ICI-contaminated OFDM systems communicating over rapidly-fading non-stationary Nakagami- $m$ channels is investigated. More specifically, we provide new and explicit analytical expressions for the PDFs of both the desired signal of each OFDM subcarrier as well as for the ICI.

The rest of this treatise is structured as follows. Section II describes the system and the channel model considered. Section III analyzes the BER performance of OFDM systems using coherent demodulation. Section IV provides our numerical results, while Section V offers our conclusions.

\section{System AND CHANNEL MODEL}

The channel is modelled by the single-path Channel Impulse Response (CIR) expressed in the Time domain (T-domain) as $h_{n}=\left|h_{n}\right| \exp \left(-j \phi_{n}\right) \triangleq r_{n} \exp \left(-j \phi_{n}\right)$. The envelope of the channel, $r_{n}=\left|h_{n}\right|$, obeys the Nakagami- $m$ distribution having a PDF given by [7]

$$
f_{r}\left(r_{n}\right)=\frac{2}{\Gamma(m)}\left(\frac{m}{\Omega}\right)^{m} r_{n}^{2 m-1} \exp \left(-\frac{m}{\Omega} r_{n}^{2}\right), \quad m \geq \frac{1}{2},
$$

where $\Gamma(\cdot)$ is the gamma function [10], $\Omega=\mathrm{E}\left[r_{n}^{2}\right]$ is the average power of the fading envelope, $E[\cdot]$ denotes the expectation and $m$ is the fading parameter. In this paper, we assume that $\Omega=1$. The joint PDF of two identically distributed Nakagami- $m$ variables is given by [7]

$$
\begin{aligned}
& f\left(r_{n}, r_{k}\right)=\frac{4}{\Gamma(m) \rho_{n k}^{(m-1) / 2}\left(1-\rho_{n k}\right)}\left(\frac{m}{\Omega}\right)^{m+1}\left(r_{n} r_{k}\right)^{m} \\
& \cdot \exp \left[-\frac{1}{1-\rho_{n k}}\left(\frac{m}{\Omega}\right)\left(r_{n}^{2}+r_{k}^{2}\right)\right] I_{m-1}\left(\frac{2 \sqrt{\rho_{n k}}}{1-\rho_{n k}}\left(\frac{m}{\Omega}\right) r_{n} r_{k}\right),
\end{aligned}
$$

where $I_{m-1}(\cdot)$ is the $(m-1)$-th order modified Bessel function of the first kind and $\rho_{n k}$ is the correlation coefficient between 
$r_{n}$ and $r_{k}$, which can be expressed as $\rho_{n k}=J_{0}^{2}\left(2 \pi f_{D}|n-k|\right)$ and $J_{0}(\cdot)$ is the zero order Bessel function of the first kind, while $f_{D}$ is the normalized maximum Doppler shift.

The Frequency domain (F-domain) symbols to be transmitted by the OFDM subcarriers are hosted by the vector $\mathbf{X}=\left[X_{0}, X_{1}, \ldots, X_{N-1}\right]^{T}$, where $N$ is the number of subcarriers and the superscript $T$ denotes the transpose of the vector. Let the received signal is expressed as $\mathbf{y}=$ $\left[y_{0}, y_{1}, \ldots, y_{N-1}\right]^{T}$. Then, if the complex-valued T-domain fading channel vector encountered by a specific OFDM symbol is $\mathbf{h}=\left[h_{0}, h_{1}, \ldots, h_{N-1}\right]$, the received signal $\mathbf{y}$ can be expressed in a matrix form as [1]

$$
\mathbf{y}=\mathcal{H} \mathbf{F}^{*} \mathbf{X}+\mathbf{w},
$$

where $\mathcal{H}$ is the diagonal T-domain channel transfer function matrix defined as $\mathcal{H}=\operatorname{diag}(\mathbf{h}), \mathbf{F}$ denotes the $N$-point Fast Fourier Transform $(N$-FFT) matrix whose $(k, i)$-th element is defined as $F_{k, i}=\frac{1}{\sqrt{N}} \exp \left(-j \frac{2 \pi}{N} k i\right)$, where we have $k, i=$ $0,1, \ldots, N-1$. Furthermore, in Eq. (3) the superscript $*$ represents the conjugate transpose and $\mathbf{w}=\left[w_{0}, w_{1}, \ldots, w_{N-1}\right]^{T}$ is the Additive White Gaussian Noise (AWGN) with zero mean and a variance of $\sigma^{2}$.

\section{BER PERFORMANCE ANALYSIS}

Assuming that the phase rotation imposed by the channel $h_{n}=\left|h_{n}\right| \exp \left(-j \phi_{n}\right), n=0,1, \ldots, N-1$, can be sufficiently accurately estimated so that coherent demodulation is possible, the signal generated in the F-domain after the FFT-based demodulation is given by

$$
\mathbf{Y}=\mathbf{F} \boldsymbol{\Phi} \mathcal{H}^{*} \mathbf{X}+\mathbf{F} \boldsymbol{\Phi} \mathbf{w}=\mathbf{F} \mathbf{H F}^{*} \mathbf{X}+\mathbf{W},
$$

where we have $\boldsymbol{\Phi}=\operatorname{diag}\left(\exp \left(-j \phi_{0}\right), \exp \left(-j \phi_{1}\right), \ldots\right.$, $\left.\exp \left(-j \phi_{N-1}\right)\right), \quad \mathbf{H}=\operatorname{diag}\left(\left|h_{0}\right|,\left|h_{1}\right|, \ldots,\left|h_{N-1}\right|\right)$ and $\mathbf{W} \triangleq \mathbf{F} \boldsymbol{\Phi} \mathbf{W}$ is the Gaussian noise in the F-domain. Then, the F-domain demodulated signal of the $k$-th subcarrier may be expressed as

$$
\begin{aligned}
& Y_{k}=X_{k} \underbrace{\left(\frac{1}{N} \sum_{n=0}^{N-1}\left|h_{n}\right|\right)}_{\alpha}+ \\
& \underbrace{\sum_{i=0, i \neq k}^{N-1} X_{i}\left(\frac{1}{N} \sum_{n=0}^{N-1}\left|h_{n}\right| \exp (-j 2 \pi n(k-i) / N)\right)}_{\beta_{k}} \\
& +\underbrace{\frac{1}{\sqrt{N}} \sum_{n=0}^{N-1} w_{n} \exp \left(-j 2 \pi n k / N+j \phi_{n}\right)}_{W_{k}} \\
& \triangleq \alpha X_{k}+\beta_{k}+W_{k} \quad k=0,1, \ldots, N-1,
\end{aligned}
$$

where $\alpha$ is the multiplicative F-domain distortion imposed on the received signal of the $k$-th subcarrier and $\beta_{k}$ is the fading-induced ICI term imposed by the loss of subcarrierorthogonality, which can be viewed as extra non-Gaussian distributed additive noise. It is readily seen that the ICI term will vanish, if the channel becomes time invariant.
In general, the PDFs of $\alpha$ and $\beta_{k}$ in Eq. (5) are required for analyzing the BER performance. However, it is non-trivial to derive the exact PDFs of $\alpha$ and $\beta_{k}$. A widely used assumption is that in case of a moderate Doppler shift, the T-domain channel variation across an OFDM symbol may be considered to be linear. To examine this assumption further, let us consider Fig. 1, where various T-domain channel envelopes associated with different Doppler shifts are presented. Fig. (1a), (2a) and (3a) portray three different T-domain channel envelopes whose normalized Doppler shifts are $f_{D}=f_{d} \cdot T_{s}=1 \times 10^{-3}$, $5 \times 10^{-3}$ and $1 \times 10^{-4}$ respectively, where $f_{d}$ is the Doppler shift in $\mathrm{Hz}$ and $T_{s}$ is the symbol duration. More explicitly, Fig. (1b), (2b) and (3b) portray three randomly chosen channel envelopes corresponding to an OFDM symbol duration, where the number of OFDM subcarriers is $N=128$. In Fig. (1b), (2b) and (3b), the solid-line represents the actual channel, while the dashed-line is the straight-line approximation of the channel. It can be seen from this figure that the lower the OFDM symbol normalized Doppler shift $f_{b}=f_{d} \cdot T_{s} \cdot N$, the better the dashed-line matches the solid-line. Let us now define the Normalized Mean Square Error (NMSE) as

$$
\mathrm{NMSE}=\mathrm{E}\left[\frac{\sum_{n}\left|h_{n}-\hat{h}_{n}\right|^{2}}{\sum_{n}\left|h_{n}\right|^{2}}\right],
$$

where $\hat{h}_{n}$ is the approximated T-domain channel, which will be used for evaluating the accuracy of the above-mentioned linear channel envelope approximation. In our simulations, the NMSE was evaluated for the three T-domain channels associated with $f_{b}=0.128,0.0128$ and 0.00128 , which was found to be $1.8 \%, 0.42 \%$ and $0.047 \%$, respectively. Therefore our conclusion is in accordance with that in [8], i.e. that the T-domain variation of the channel envelope across an OFDM symbol can indeed be modelled as a straight line, when we have $f_{b}<0.1$.
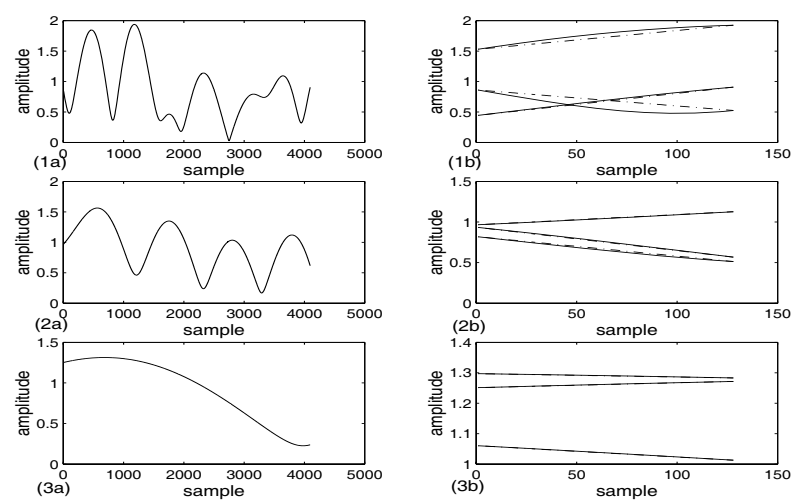

Fig. 1. T-domain channels using $m=1, N=128$ in conjunction with $f_{D}=1 \times 10^{-3}$ for (1a) and (1b), $f_{D}=5 \times 10^{-4}$ for (2a) and (2b), $f_{D}=$ $1 \times 10^{-4}$ for (3a) and (3b).

Based on the above-mentioned assumption, $\alpha$ in Eq. (5) can be simplified to the scaled sum of two correlated Nakagami variables

$$
\alpha=\frac{1}{N} \sum_{n=0}^{N-1}\left|h_{n}\right|=\frac{1}{2}\left(\left|h_{0}\right|+\left|h_{N-1}\right|\right) \triangleq \frac{1}{2}\left(r_{0}+r_{N-1}\right) .
$$


Since the joint PDF of two identically distributed Nakagami- $m$ variables was given by Eq. (2), the PDF of $\alpha$ can be expressed as [9]

$$
f_{\alpha}(\alpha)=2 \int_{0}^{2 \alpha} f\left(2 \alpha-r_{N-1}, r_{N-1}\right) d r_{N-1}
$$

where based on Eq. (2) we have

$$
\begin{aligned}
& f\left(2 \alpha-r_{N-1}, r_{N-1}\right)=\frac{4}{\Gamma(m) \rho_{0(N-1)}^{(m-1) / 2}\left(1-\rho_{0(N-1)}\right)}\left(\frac{m}{\Omega}\right) \\
& \cdot\left[\left(2 \alpha-r_{N-1}\right) r_{N-1}\right]^{m} \exp \left(-\frac{1}{1-\rho_{0(N-1)}}\left(\frac{m}{\Omega}\right)\left[\left(2 \alpha-r_{N}\right.\right.\right. \\
& \left.\left.+r_{2}^{2}\right] \cdot\right) I_{m-1}\left(\frac{2 \sqrt{\rho_{0(N-1)}}}{1-\rho_{0(N-1)}}\left(\frac{m}{\Omega}\right)\left(2 \alpha-r_{N-1}\right) r_{N-1}\right) .
\end{aligned}
$$

\section{A. BER based on Gaussian-distributed ICI assumption}

There is a widely used assumption that, when $N$ is large, the ICI term $\beta_{k}$ in Eq. (5) can be approximated by a Gaussian distributed noise process having zero mean [3], [4]. Since the power of the ICI is the power leaks to subcarriers other than the desired subcarrier, so the variance of the ICI can be measured by the difference between the total signal power and the desired signal power. The desired signal power of the $k$-th subcarrier is given by $\mathrm{E}\left[\left|\alpha X_{k}\right|^{2}\right]=E_{s} \cdot \mathrm{E}\left[\alpha^{2}\right]$, where $E_{s}$ is the symbol energy. The derivation of the expectation $\mathrm{E}\left[\alpha^{2}\right]$ of $\alpha^{2}$ can be found in the Appendix, yielding:

$$
\begin{aligned}
& \mathrm{E}\left[\alpha^{2}\right]=\mathrm{E}\left[\left(\frac{1}{N} \sum_{n=0}^{N-1}\left|h_{n}\right|\right)\left(\frac{1}{N} \sum_{k=0}^{N-1}\left|h_{k}\right|\right)\right] \\
& =\frac{1}{N^{2}} \sum_{n=0}^{N-1} \sum_{k=0}^{N-1} \int_{0}^{\infty} \int_{0}^{\infty} r_{n} r_{k} p\left(r_{n}, r_{k}\right) d r_{n} d r_{k} \\
& =\frac{1}{N^{2}}\left(N+\frac{2 \Gamma^{2}\left(m+\frac{1}{2}\right)}{\Gamma^{2}(m)}\left(\frac{\Omega}{m}\right) \sum_{i=1}^{N-1}(N-i)\right. \\
& \left.{ }_{2} \mathrm{~F}_{1}\left(-\frac{1}{2},-\frac{1}{2} ; m ; \rho_{n k}\right)\right),
\end{aligned}
$$

where ${ }_{2} \mathrm{~F}_{1}($.$) is the hypergeometric function [10]. Therefore$ the variance of the ICI of the $k$-th subcarrier can be derived as

$$
\begin{aligned}
& \sigma_{\beta_{k}}^{2}=E_{s}\left(\mathrm{E}\left[\frac{1}{N} \sum_{n=0}^{N-1}\left|h_{n}\right|^{2}\right]-\mathrm{E}\left[\alpha^{2}\right]\right) \\
& =E_{s}\left\{1-\frac{1}{N^{2}}\left(N+\frac{2 \Gamma^{2}\left(m+\frac{1}{2}\right)}{\Gamma^{2}(m)}\left(\frac{\Omega}{m}\right) \sum_{i=1}^{N-1}(N-i)\right.\right. \\
& \left.\left.\cdot{ }_{2} \mathrm{~F}_{1}\left(-\frac{1}{2},-\frac{1}{2} ; m ; \rho_{n k}\right)\right)\right\} \triangleq E_{s} \mu
\end{aligned}
$$

Based on this Gaussian-distributed ICI assumption, the BER of the ICI-contaminated OFDM system using BPSK where $Q(x)$ is the Gaussian Q-function generally defined as $Q(x)=(1 / \sqrt{2 \pi}) \int_{x}^{\infty} e^{-t^{2} / 2} d t, \gamma=\sigma^{2} / E_{s}$ is the noise-tosignal ratio and $f\left(2 \alpha-r_{N-1}, r_{N-1}\right)$ is given by Eq. (9).

modulation can be formulated as

$$
\begin{aligned}
& P_{e}=\int_{0}^{+\infty} Q\left(\sqrt{\frac{E_{s} \alpha^{2}}{\sigma_{\beta_{k}}^{2}+\sigma^{2}}}\right) f_{\alpha}(\alpha) d \alpha=\int_{0}^{+\infty} \\
& Q\left(\sqrt{\frac{E_{s}}{E_{s} \mu+\sigma^{2}}} \alpha\right) \cdot 2 \int_{0}^{2 \alpha} f\left(2 \alpha-r_{N-1}, r_{N-1}\right) d r_{N-1} d \alpha \\
& =2 \int_{0}^{+\infty} \int_{0}^{2 \alpha} Q\left(\frac{1}{\sqrt{\mu+\gamma}} \alpha\right) f\left(2 \alpha-r_{N-1}, r_{N-1}\right) d r_{N-1} d \alpha
\end{aligned}
$$

\section{B. BER based on proposed analytic PDF of ICI}

Although the above-mentioned assumption of Gaussiandistributed ICI is widely used, below we will demonstrate that this assumption is inaccurate and results in a pessimistic BER estimate, more specifically, exhibiting an error floor. In order to analyze the PDF of the ICI, we rewrite Eq. (4) (ignoring the AWGN) as

$$
\mathbf{Y}=\mathbf{F H}_{s} \mathbf{F}^{*} \mathbf{X}+\mathbf{F H}_{c} \mathbf{F}^{*} \mathbf{X}
$$

where $\mathbf{H}_{s}$ is a diagonal matrix whose diagonal elements are equal to $\frac{\left|h_{0}\right|+\left|h_{N-1}\right|}{2}$ and $\mathbf{H}_{c}=\operatorname{diag}\left(-\frac{\left|h_{N-1}\right|-\left|h_{0}\right|}{2}\right.$, $\left.-\frac{(N-3)\left(\left|h_{N-1}\right|-\left|h_{0}\right|\right)^{2}}{2(N-1)}, \ldots, \frac{\left|h_{N-1}\right|-\left|h_{0}\right|}{2}\right)$. It is plausible that the second term in Eq. (13) describes the ICI. Letting $\bar{h} \triangleq \frac{\left|h_{N-1}\right|-\left|h_{0}\right|}{2}$, the second term in Eq. (13) representing the ICI can be expressed as $\mathbf{C}=\mathbf{F} \cdot \operatorname{diag}\left(-\bar{h},-\frac{N-3}{N-1} \bar{h}, \ldots, \frac{N-3}{N-1} \bar{h}, \bar{h}\right) \cdot \mathbf{F}^{*} \mathbf{X}$. For the $k$-th OFDM subcarrier, the ICI contamination may be expressed from $\mathbf{C}$ as

$$
\begin{aligned}
\beta_{k} & =\sum_{i=0}^{N-1} X_{i}\left(\sum_{n=0}^{N-1} F_{k, n} \frac{2 n-(N-1)}{N-1} \bar{h} F_{i, n}^{*}\right) \\
& =\frac{\bar{h}}{N} \sum_{i=0}^{N-1} X_{i}\left(\sum_{n=0}^{N-1}\left(\frac{2 n}{N-1}-1\right) \exp \left(j \frac{2 \pi}{N} n(i-k)\right)\right) \\
& \triangleq \frac{\bar{h}}{N} \sum_{i=0}^{N-1} X_{i} c_{k, i} \quad k=0,1, \ldots, N-1
\end{aligned}
$$

where $c_{k, i}$ can be formulated as

$$
\begin{aligned}
& c_{k, i}=\frac{2}{N-1} \sum_{n=0}^{N-1} n \exp \left(j \frac{2 \pi}{N} n(i-k)\right) \\
& -\sum_{n=0}^{N-1} \exp \left(j \frac{2 \pi}{N} n(i-k)\right) \triangleq \frac{2}{N-1} a-b . \\
& \quad k=0,1, \ldots, N-1, \quad i=0,1, \ldots, N-1 .
\end{aligned}
$$

It can be shown that in Eq. (15) we have

$$
\begin{aligned}
& a=\left\{\begin{array}{l}
-N / 2, \quad i \neq k \\
N(N-1) / 2, \quad i=k
\end{array}\right. \\
& b=\left\{\begin{array}{l}
0, \quad i \neq k \\
N, \quad i=k
\end{array}\right.
\end{aligned}
$$


therefore

$c_{k, i}=\left\{\begin{array}{l}-N /(N-1), \quad i=0,1, \ldots, N-1 \quad \text { and } \quad i \neq k \\ 0, \quad i=k\end{array}\right.$

Now substituting Eq. (17) into Eq. (14), the ICI of the $k$-th OFDM subcarrier becomes

$$
\beta_{k}=-\frac{\bar{h}}{N-1} \sum_{i=0, i \neq k}^{N-1} X_{i}
$$

It is clear that the symbol transmitted at the desired subcarrier has no contribution to the ICI of this subcarrier, while the symbols transmitted at all other subcarriers will contribute to the ICI of this subcarrier.

We assume that BPSK modulation is used (but also note that other modulation schemes may be analyzed in a similar fashion), i.e. we have $X_{i}=+1$ or -1 with equal probability. We note that the sum, $z \triangleq \sum_{i=0, i \neq k}^{N-1} X_{i}$, in Eq. (18) is binomial distributed with a density of [11]

$$
\begin{aligned}
p_{z}(z) & =\left(\begin{array}{c}
N-1 \\
\frac{z+N-1}{2}
\end{array}\right) \cdot 2^{1-N}, \\
z & \in\{1-N, 3-N, \ldots, N-3, N-1\} .
\end{aligned}
$$

Following from Eq. (18) the ICI of the $k$-th OFDM subcarrier becomes

$$
\beta_{k}=-\frac{z \bar{h}}{N-1}=-\frac{z\left(\left|h_{N-1}\right|-\left|h_{0}\right|\right)}{2(N-1)}=-\frac{z \epsilon}{2(N-1)},
$$

where $\epsilon \triangleq\left|h_{N-1}\right|-\left|h_{0}\right|=r_{N-1}-r_{0}$ is the difference of two correlated Nakagami- $m$ variables. More explicitly, it can be seen from Eq. (20) that the ICI can be modelled as a function of the difference of two correlated Nakagami-m variables. Noting that the ICI is independent of the specific subcarrier index, the subscript $k$ in $\beta_{k}$ may be omitted in the ICI-related terms. Invoking Eq. (2), we have [9] $f_{\epsilon}(\epsilon)=$ $\int_{0}^{+\infty} f\left(r_{0}, r_{0}+\epsilon\right) d r_{0}$. Noting that in Eq. (20) $z$ and $\epsilon$ are independent, therefore the PDF of the ICI can be derived as [9]

$$
\begin{aligned}
& f_{\beta}(\beta)=\sum_{z} \frac{2(N-1)}{|z|} p_{z}(z) f_{\epsilon}\left(\frac{2(1-N) \beta}{z}\right) \\
& =\sum_{z} \frac{2(N-1)}{|z|} p_{z}(z) \int_{0}^{+\infty} f\left(r_{0}, r_{0}+\frac{2(1-N) \beta}{z}\right) d r_{0} \\
& \quad z \in\{1-N, 3-N, \ldots, N-3, N-1\}
\end{aligned}
$$

where based on Eq. (2) we have

$$
\begin{aligned}
& f\left(r_{0}, r_{0}+\frac{2(1-N) \beta}{z}\right)=\frac{4}{\Gamma(m) \rho_{0(N-1)}^{(m-1) / 2}\left(1-\rho_{0(N-1)}\right)} \\
& \left(\frac{m}{\Omega}\right)^{m+1}\left[r_{0}\left(r_{0}+\frac{2(1-N) \beta}{z}\right)\right]^{m} \\
& \exp \left(-\frac{1}{1-\rho_{0(N-1)}}\left(\frac{m}{\Omega}\right)\left[r_{0}^{2}+\left(r_{0}+\frac{2(1-N) \beta}{z}\right)^{2}\right]\right) \\
& I_{m-1}\left(\frac{2 \sqrt{\rho_{0(N-1)}}}{1-\rho_{0(N-1)}}\left(\frac{m}{\Omega}\right) r_{0}\left(r_{0}+\frac{2(1-N) \beta}{z}\right)\right) .
\end{aligned}
$$

Based on Eq. (9), Eq. (22) and the PDF of the AWGN, the BER of the ICI-contaminated OFDM system using BPSK for transmission over Nakagami- $m$ fading channels can be expressed as

$$
\begin{aligned}
& P_{b e r}=\int_{-\infty}^{+\infty} \int_{-\infty}^{+\infty} \int_{0}^{+\infty} Q\left(\sqrt{\frac{E_{s} \alpha^{2}}{(\beta+W)^{2}}}\right) f_{\alpha}(\alpha) f_{\beta}(\beta) \\
& \cdot f_{W}(W) d \alpha d \beta d W=\frac{1}{\sqrt{2 \pi} \sigma} \int_{-\infty}^{+\infty} \int_{-\infty}^{+\infty} \int_{0}^{+\infty} Q\left(\frac{\sqrt{E_{s}} \alpha}{|\beta+W|}\right) \\
& \exp \left(-\frac{W^{2}}{2 \sigma^{2}}\right) f_{\alpha}(\alpha) f_{\beta}(\beta) d \alpha d \beta d W
\end{aligned}
$$

where $f_{\alpha}(\alpha)$ and $f_{\beta}(\beta)$ are given in (8) and (21) respectively.

\section{Numerical RESUlts}

Numerical experiments have been conducted for evaluating the BER performance of ICI-contaminated OFDM systems using the proposed method. The number of subcarriers was $N=128$, the maximum Doppler shift was $f_{d}=100 \mathrm{~Hz}$ and the data rate was assumed to be $200 \mathrm{KBit} / \mathrm{s}$.

Fig. 2 shows our BER results as a function of the $E_{b} / N_{0}$ value for the proposed BER evaluation method, when different values of $m$ are used. Below we will provide a range of results, when the Nakagami fading parameter is set to $m=1$ and the Jakes' channel model [12] was used for generating the T-domain Rayleigh-fading channel.

Fig. 3 demonstrates the differences amongst the various ICI PDFs, when different ICI-modelling techniques are used. It can be seen that the true ICI PDF generated by simulation is significantly narrower than the ICI PDF based on the Gaussian approximation, but quite close to the proposed ICI PDF. Since a higher ICI will result in a higher BER, we can predict that the BER performance derived using the Gaussian approximation of the ICI will be significantly worse than the true BER.

Fig. 4 presents our BER results as a function of the $E_{b} / N_{0}$ value for the different BER evaluation methods considered. The BER results of the Gaussian ICI approximation method were calculated according to Eq. (12). The results of the proposed analytical method were evaluated from Eq. (23). The results of the ICI-free OFDM systems were calculated according to Eq. (12) but $\mu$ was set to zero. It is clear that our proposed analytical ICI-modelling technique provides a good approximation of the simulated BER, while the Gaussian ICI approximation technique presented pessimistic results. In fact, the Gaussian ICI approximation technique and the zeroICI scenario constitute the upper-bound and lower-bound BER limits for the proposed method respectively.

\section{CONCLUSIONS}

The BER performance of ICI-contaminated OFDM systems using coherent demodulation was analyzed when communicating over instantaneously fading non-dispersive Nakagami$m$ channels. Based on the joint distribution of two correlated Nakagami- $m$ variables, we provided explicit analytical expressions for both the PDF of the desired signal and the PDF of the ICI. Our simulation results validate our analysis, suggesting that the widely used Gaussian approximation of the ICI is inaccurate and hence results in a pessimistic BER estimate. By contrast, the proposed method matches the simulation results 
significantly more closely. Our future research will consider the analysis of multilevel modulation schemes.

\section{APPENDIX}

First, the two-dimension (2-D) integral seen in Eq. (10) is derived. Using Eqs. (2), (8.406.3), Eq. (6.631.1) and Eqs. (7.621.4), (9.131.1) in [10], the 2-D integral can be written as

$$
\begin{aligned}
& \int_{0}^{\infty} \int_{0}^{\infty} r_{n} r_{k} p\left(r_{n}, r_{k}\right) d r_{n} d r_{k}=\frac{2\left(1-\rho_{n k}\right)^{1 / 2} \Gamma\left(m+\frac{1}{2}\right)}{\Gamma^{2}(m)} \\
& \cdot\left(\frac{m}{\Omega}\right)^{m-\frac{1}{2}} \int_{0}^{\infty} r_{k}^{2 m} \exp \left(-\frac{1}{1-\rho_{n k}}\left(\frac{m}{\Omega}\right) r_{k}^{2}\right) \\
& { }_{1} \mathrm{~F}_{1}\left(m+\frac{1}{2}, m ; \frac{\rho_{n k}}{1-\rho_{n k}}\left(\frac{m}{\Omega}\right) r_{k}^{2}\right) d r_{k} \\
& =\frac{\Gamma^{2}\left(m+\frac{1}{2}\right)}{\Gamma^{2}(m)}\left(\frac{m}{\Omega}\right)^{-1}{ }_{2} \mathrm{~F}_{1}\left(-\frac{1}{2},-\frac{1}{2} ; m ; \rho_{n k}\right)
\end{aligned}
$$

Substituting Eq. (24) into Eq. (10), E $\left[\alpha^{2}\right]$ can be expressed as

$$
\begin{aligned}
& \mathrm{E}\left[\alpha^{2}\right]=\frac{1}{N^{2}} \sum_{n=0}^{N-1} \sum_{k=0}^{N-1} \frac{\Gamma^{2}\left(m+\frac{1}{2}\right)}{\Gamma^{2}(m)}\left(\frac{m}{\Omega}\right)^{-1} \\
& { }_{2} \mathrm{~F}_{1}\left(-\frac{1}{2},-\frac{1}{2} ; m ; \rho_{n k}\right)=\frac{1}{N^{2}}\left(N+\frac{2 \Gamma^{2}\left(m+\frac{1}{2}\right)}{\Gamma^{2}(m)}\left(\frac{\Omega}{m}\right)\right. \\
& \left.\sum_{i=1}^{N-1}(N-i)_{2} \mathrm{~F}_{1}\left(-\frac{1}{2},-\frac{1}{2} ; m ; \rho_{n k}\right)\right) .
\end{aligned}
$$

\section{REFERENCES}

[1] L. Hanzo, M. Munster, B. J. Choi and T. Keller, OFDM and MC-CDMA for broadband multi-user communications, WLANs and Broadcasting, John Wiley \& Sons, IEEE Press, 2003.

[2] L. J. Cimini, Jr., "Analysis and simulation of a digital mobile channel using orthogonal frequency division multiplexing," IEEE Transactions on Communications, vol. 33, no. 7, pp. 665-675, July 1985.

[3] G. L. Stüber, Principles of mobile communication, 2nd Edition, Springer, 2000.

[4] Y. H. Kim, I. Song, H. G. Kim, T. Chang and H. M. Kim, "Performance analysis of a coded OFDM system in time-varying multipath Rayleigh fading channels," IEEE Transanctions on Vehicular Technology, vol. 48, no. 5, pp. 1610-1615, Sep. 1999.

[5] I. Bradaric and A. P. Petropulu, "Performance of training-based OFDM systems in the presence of time varying frequency-selective channels," in Proceedings of IEEE International Conference on Acoustic, Speech and Signal Processing, ICASSP 2004, pp. 737-740.

[6] T. Wang, J. G. Proakis and J. R. Zeidler, "Performance analysis of high QAM OFDM system over frequency selective time-vaving fading channel," in Proceedings of IEEE International Symposium on Personal, Indoor and Mobile Radio Communications, PIMRC 2003, pp.793-798.

[7] M. Nakagami, "The $m$-distribution - A general formula of intensity distribution of rapid fading," in Statistical Methods in Radio Wave Propagation, W. G. Hoffman, Ed. Oxford, U.K.: Pergamon, 1960.

[8] W. G. Jeon, K. H. Chang, and Y. S. Cho, "An equalization technique for orthogonal frequency-division multiplexing systems in time-variant multipath channels," IEEE Transanctions on Communications, vol. 47, no. 1, pp. 27-32, Jan. 1999.

[9] A. Papoulis, Probability, Random Variables, and Stochastic Processes, 3rd Edition, New York: McGraw-Hill, 1991

[10] I. S. Gradshteyn and I. M. Ryzhik, Table of integrals, series and products, 4th Edition, Academic Press, 1965.

[11] R. K. Morrow, JR., and J. S. Lehnert, "Bit-to-bit error dependence in slotted DS/SSMA packet systems with random signature sequences," IEEE Transanctions on Communications, vol. 37, no. 10, pp. 1052-1061, Oct. 1989

[12] W. C. Jakes Jr., Ed., Microwave Mobile Communications. New York: Wiley, 1974.

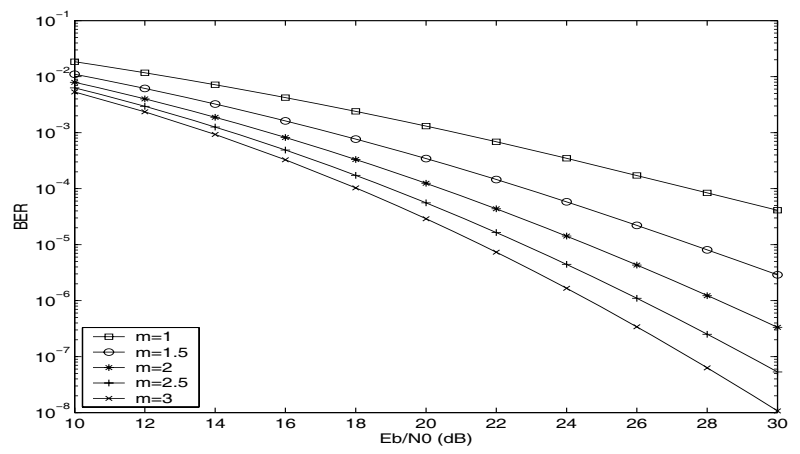

Fig. 2. BER versus $E_{b} / N_{0}$ performance of ICI-contaminated BPSKmodulated OFDM over Nakagami- $m$ fading channels using $f_{D}=5 \times 10^{-4}$ and $N=128$ subcarriers.

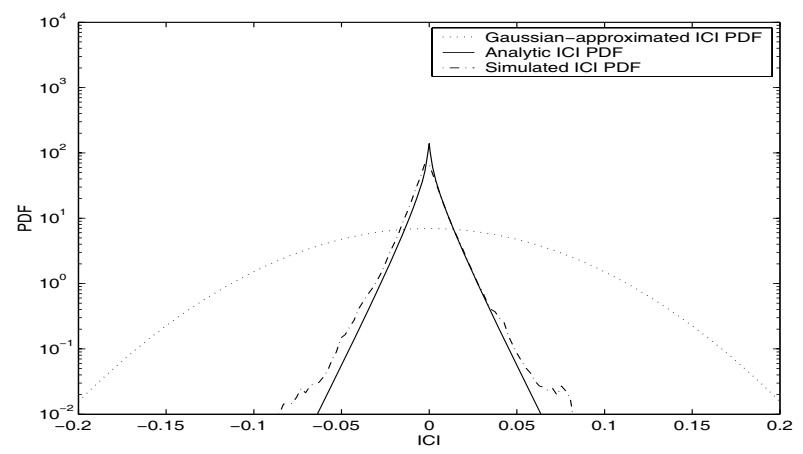

Fig. 3. PDF of ICI using $m=1, N=128$ and $f_{D}=5 \times 10^{-4}$.

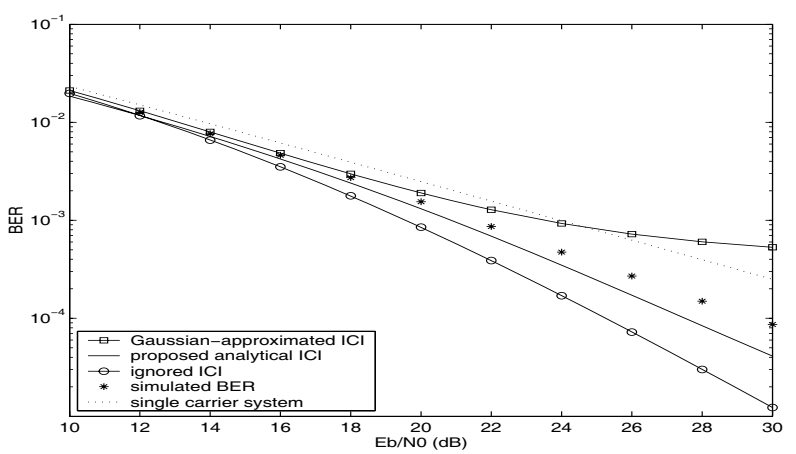

Fig. 4. BER versus $E_{b} / N_{0}$ performance of ICI-contaminated BPSKmodulated OFDM over Nakagami fading channels using $m=1, f_{D}=$ $5 \times 10^{-4}$ and $N=128$ subcarriers. 